\title{
Difficult Diagnosis and Differentiated Treatment in Synchronous Thoracic Hemangiomas
}

\author{
Viorica Vidul $^{1}$, Vasile Popa ${ }^{2}$, Genevieve Wu \\ 1. Center for General Surgery and Liver Transplantation, Clinical Institute of Digestive Diseases and \\ Liver Transplant, Fundeni, Bucharest 2. Thoracic Surgery Department, Emergency Clinical Hospital, \\ "Sfântul Ioan", Bucharest, Romania 3.
}

$\square$ Corresponding author: Valentin M. Munteanu, munteanuvalentin@yahoo.com Disclosures can be found in Additional Information at the end of the article

\section{Abstract}

Thoracic hemangiomas are rare vascular neoplasia among adults. Even with advanced imaging, diagnosis is sometimes impossible, thereby mandating histopathological examination. The differential diagnosis of thoracic hemangiomas can include arteriovenous malformations, thoracic sarcomas (angiosarcoma, paravertebral mesothelioma), neurinoma, and neurofibroma. We herein present a case which was intitially suggestive of a malignancy that ultimately proved to be a capillary cavernous paravertebral and vertebral hemangioma. Given the unique topography of this lesion, a correct preoperative diagnosis was not possible and a interdisciplinary surgical approach was required.

Categories: Cardiac/Thoracic/Vascular Surgery, General Surgery, Neurosurgery

Keywords: tumor, capillary-cavernous hemangioma, vertebral and paravertebral, surgical treatment

\section{Introduction}

Considered a relatively rare disease in adult pathology, hemangiomas represent a controversial entity in terms of their pathogenic, clinical and therapeutic characteristics. The nosologic framework of hemangioma continues to be debated, having been defined in the past as either congenital vascular malformations (hamartomas) or benign vascular malignancies with a locally destructive nature. In an adult population, hemangiomas, especially the cavernous type, are located in bones, especially the vertebrae, thorax, brain, liver, and skeletal muscle. Since the clinical and imaging apperance of hemangioma can mimic a malignant tumor, histopathological examination is frequently required.

Because there is no universally accepted standard treatment of hemangiomas, several therapeutic alternatives are generally considered: surgery, embolization, direct injection of

\section{Published 02/10/2011}

\section{(c) Copyright 2011}

Vidul et al. This is an open access article distributed under the terms of the Creative Commons Attribution License CC-BY 3.0., which permits unrestricted use, distribution, and reproduction in any medium, provided the original author and source are credited. ethanol (alcohol), chemotherapy, radiotherapy, corticosteroids, immunotherapy depending on age, topography, histopathological type, complications, and local destructive nature. In about $10 \%$ of cases, hemangiomas are synchronous. In these situations, the differential diagnosis is even more difficult; it is imperative to rule out malignancy. Ultimately, treatment varies depending on topography and the evolutionary stage of the lesion.

Since hemangiomas are relatively rare entities, with especially misleading clinical and imaging characteristics, their management can be challenging. The case we present herein both illustrates these difficulties as well as demonstrates the value of interdisciplinary collaboration. 


\section{Cureus}

\section{Case Presentation}

This 45-year-old male patient, without a significant antecedent history of disease, presented with a several year history of progressively severe chest pain that responded poorly to a range of pain medications. Despite his severe chest pain, physical examination was grossly normal. However, chest computed tomography (CT) revealed a solid tumor located in the left paravertebral muscles, adhering to the parietal pleura. Imaging was suggestive for a paravertebral mesothelioma or muscle sarcoma.

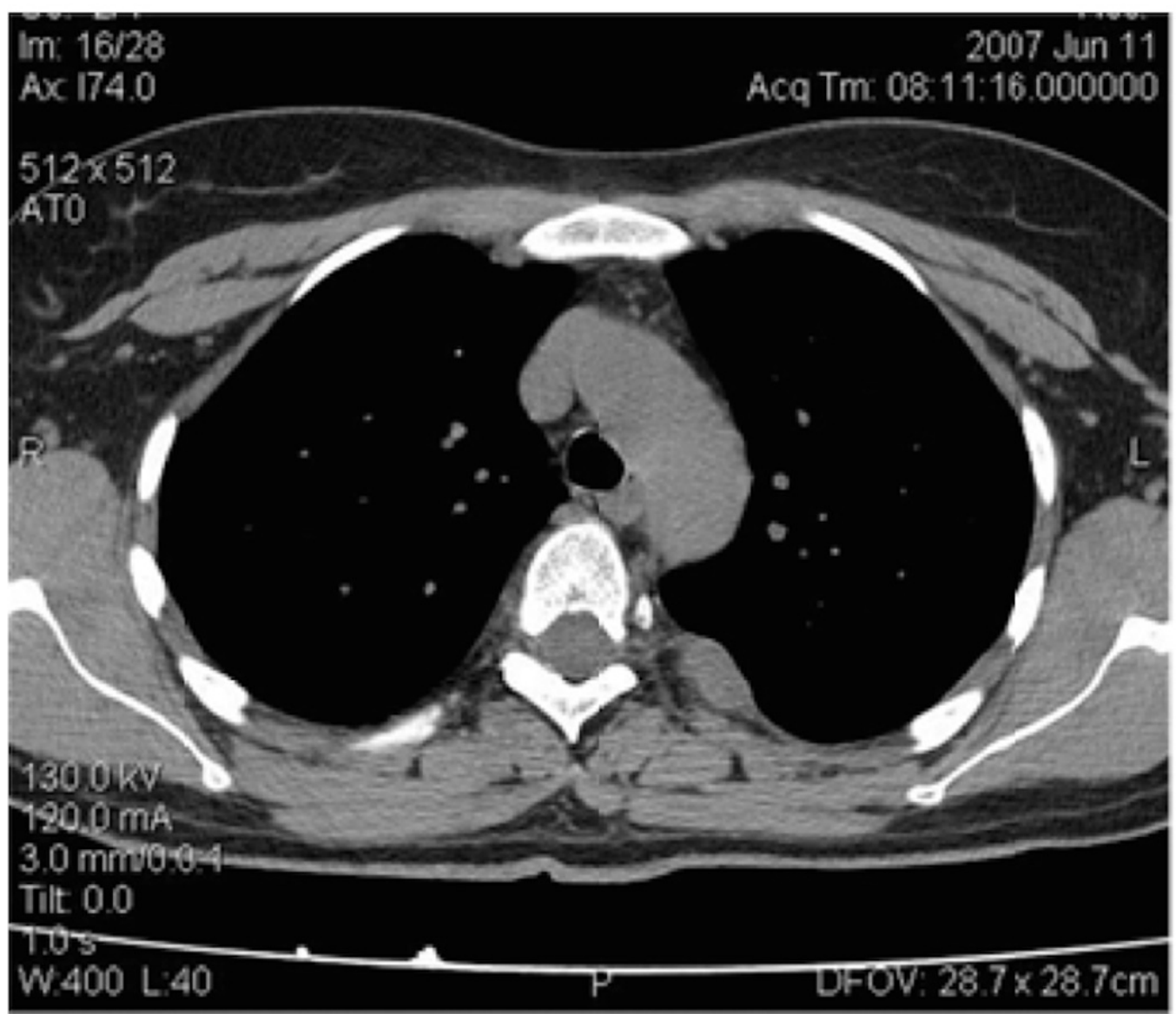

FIGURE 1: Aspect of chest computer tomography: left paravertebral tumor

Within the posterior mediastinum, a contrast magnetic resonance imaging (MRI) revealed a left paravertebral tumor of $5.5 \mathrm{~cm}$, located in the V-VI intercostal space. The lesion was extrapleural in location, polilobar in shape and both hyperintense on T2-weighted and hypointense on T1- weighted MRI. Furthermore, imaging demonstrated the mass to be inhomogeneous, well-vascularized, and without invasion or destruction of the adjacent ribs or neural foramen (Figure $2 A, 2 B$ ). 


\section{Cureus}
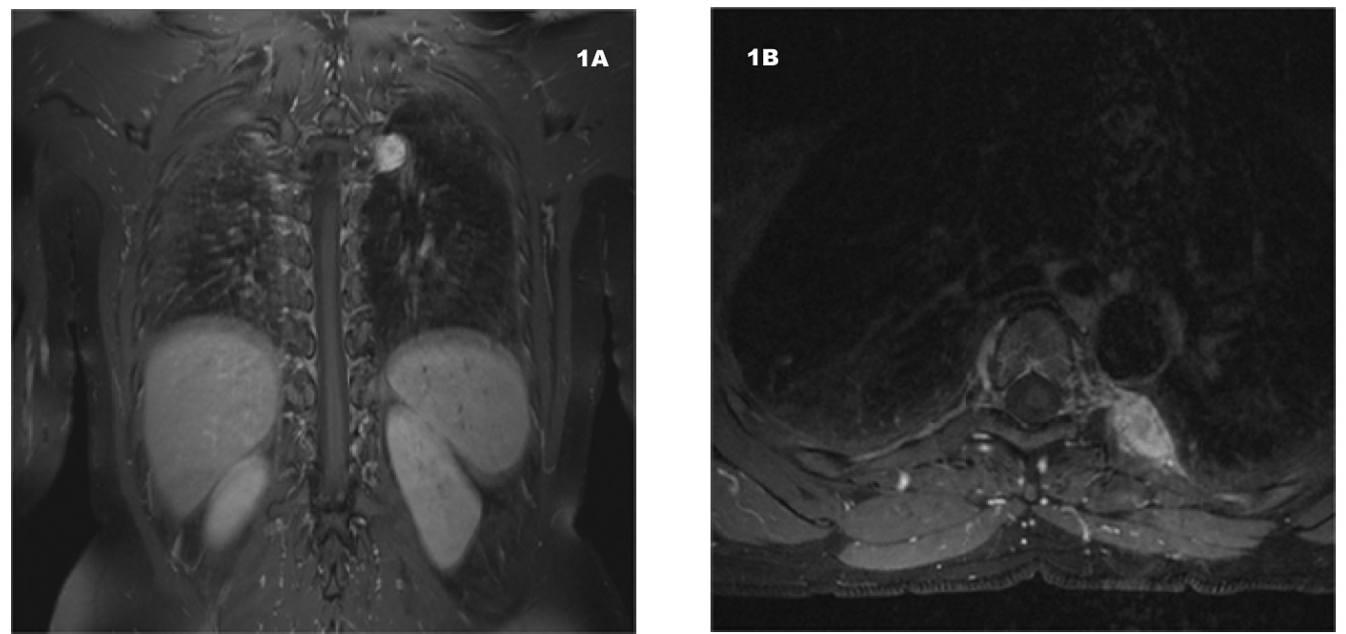

FIGURE 2: Aspect of magnetic resonance with contrast study: paravertebral and intercostals T5-6 tumor

A. Sagital image B. Transversal image a level T5-T6;

The appearance was most consistent with an intercostal nerve sheath tumor. Meanwhile, a second lesion was seen on MRI adjacent to and contacting the T6 vertebral body, but without violating the integrity of cortical bone (Figure $3 A, 3 B$ ).
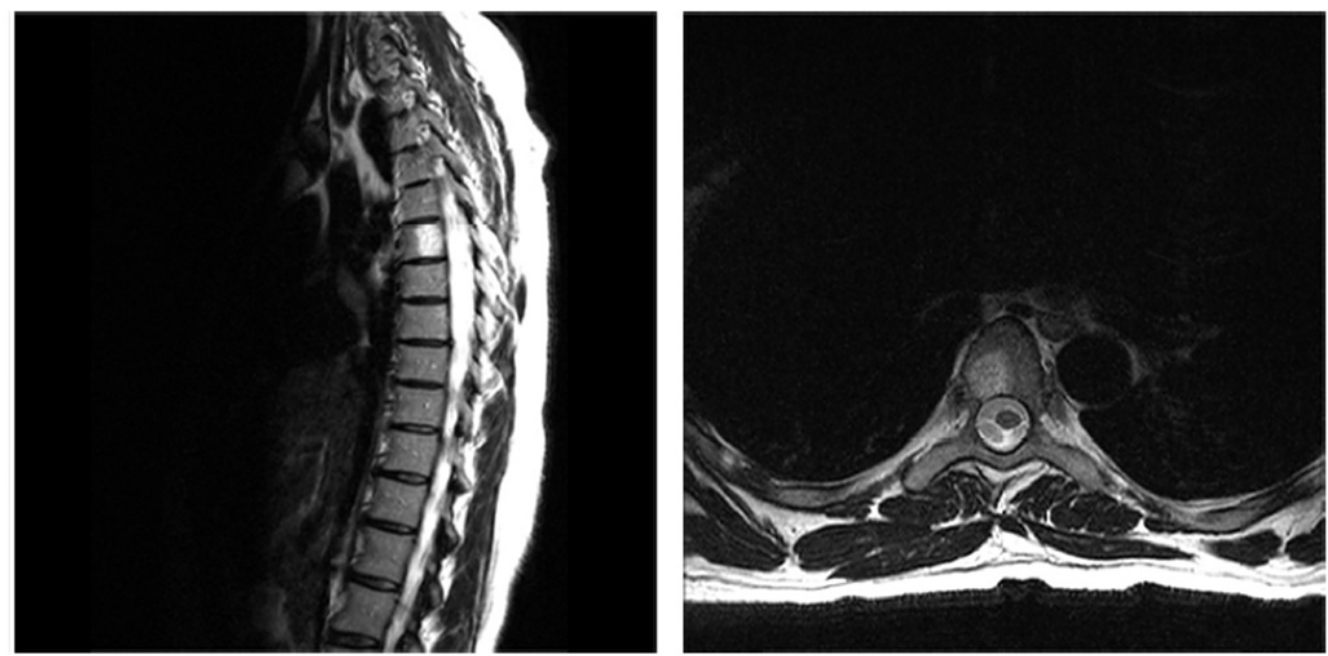

FIGURE 3: Aspect of magnetic resonance imaging with angiographic study: vertebral hemangioma T6 with cortical bone limits $(A, B)$

A. Sagital image B. Transversal image a level T6 


\section{Cureus}

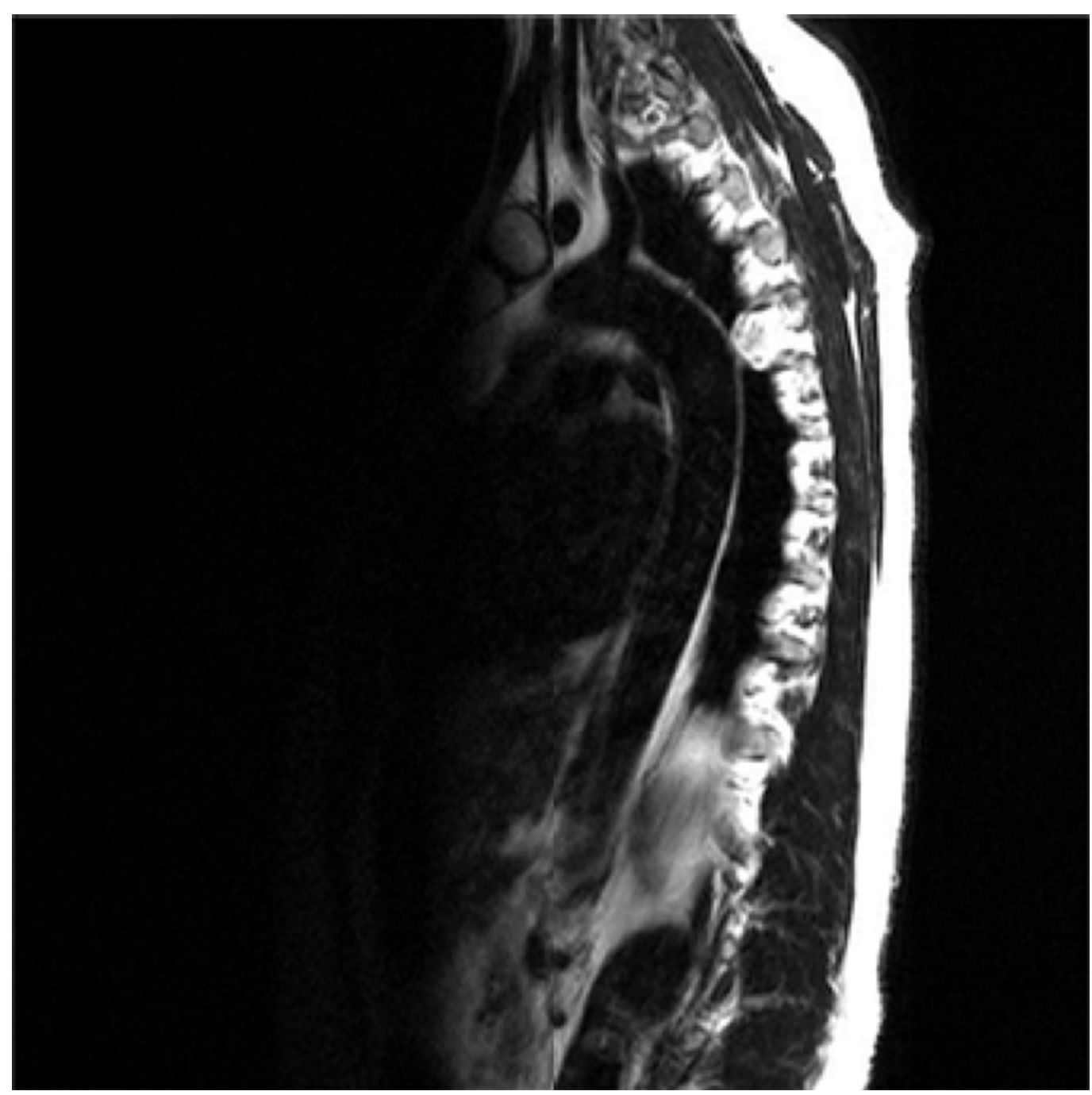

FIGURE 4: Aspect of magnetic resonance imaging with angiographic study: paravertebral tumor with vertebral T6 contact.

Given a presumptive diagnosis of an intercostal neurinoma, but also considering the possibility of a paravertebral mesothelioma, the patient underwent surgery by the Thoracic Surgery Department of the St. John's Hospital. A left axillary thoracotomy with sectioning of the latissimus dorsi and serratus muscles was performed. A purple, partially encapsulated polilobar lesion was found intraoperatively located in the intercostal space, which was attached to a thoracic spine vascular/nervous pedicle. The neural foramen was closed and the tumor was found to have destroyed the adjacent intercostal muscle (Figure 5). 


\section{Cureus}

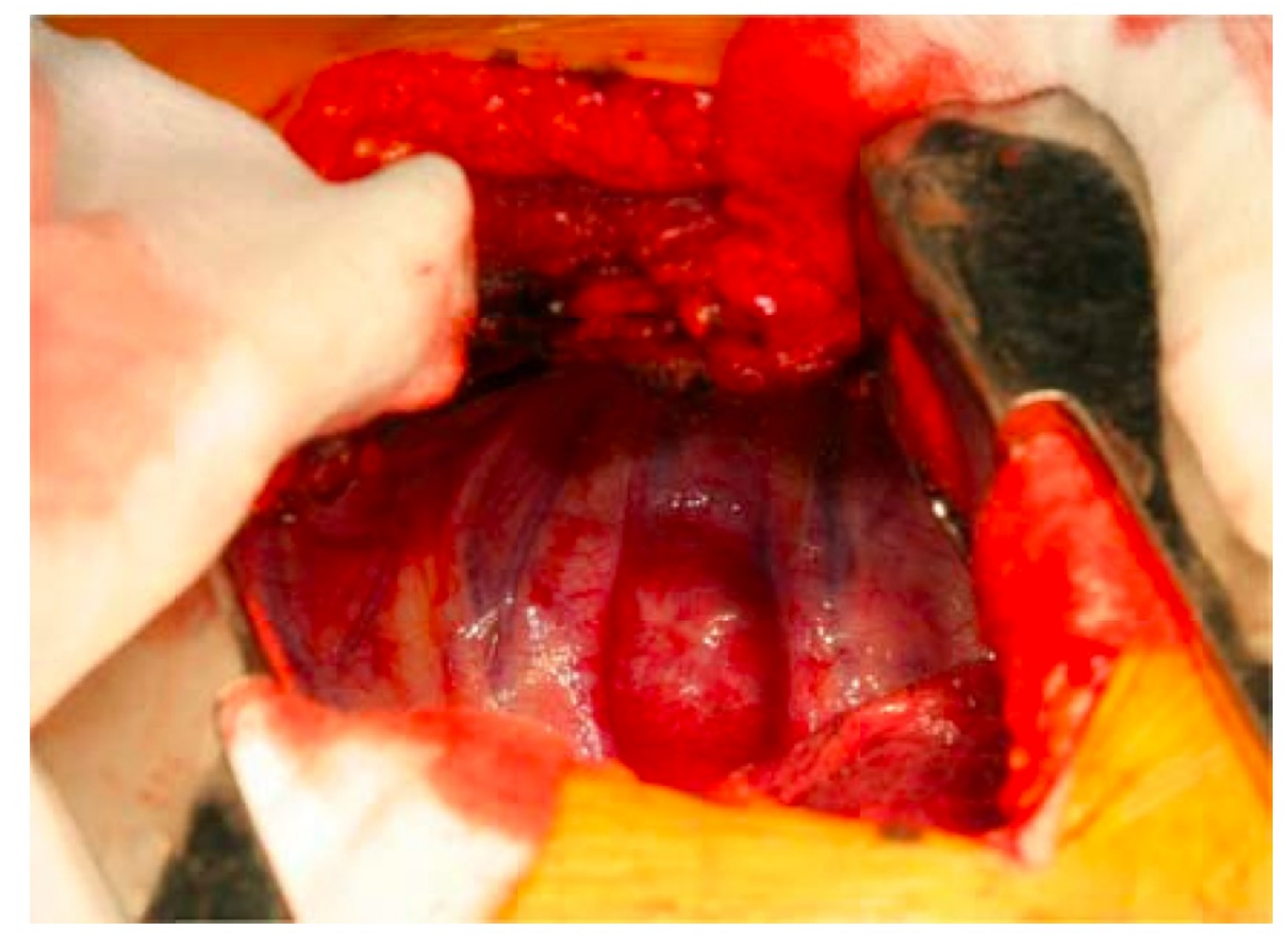

FIGURE 5: Intraoperative appearance of the vertebral capillary cavernous hemangioma with intra-thoracic extension.

We performed a en-block resection of the lesion, including the parietal pleura of the thoracic spine and the remaining intercostal muscles. Because of serious intraoperative hemorrhage, it proved necessary to dissect, ligate and section the posterior intercostal artery (Figure 6). Parietal plural and paravertebral hemostasis was achieved with TachoSil (Figure 7). 


\section{Cureus}

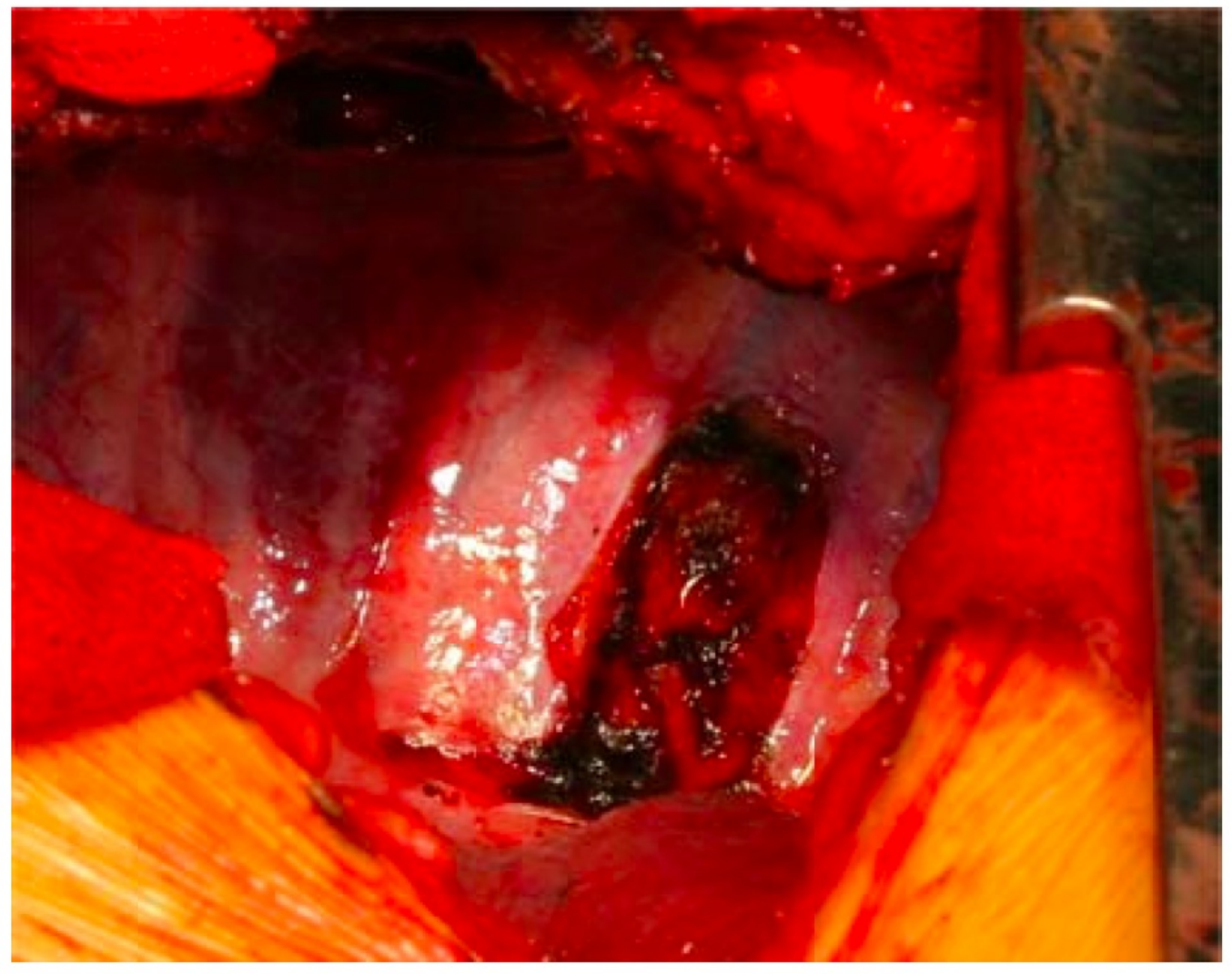

FIGURE 6: Intraoperative aspect after paravertebral tumor and parietal pleura excision

Intraoperative aspect after paravertebral tumor and parietal pleura excision, with intercostal muscles and artery excision, after dissection, ligature and section anterior vertebral artery and dissection paravertebral simpatic lymph nodes at T5-T6 level. Foramen ovale is closed. 


\section{Cureus}

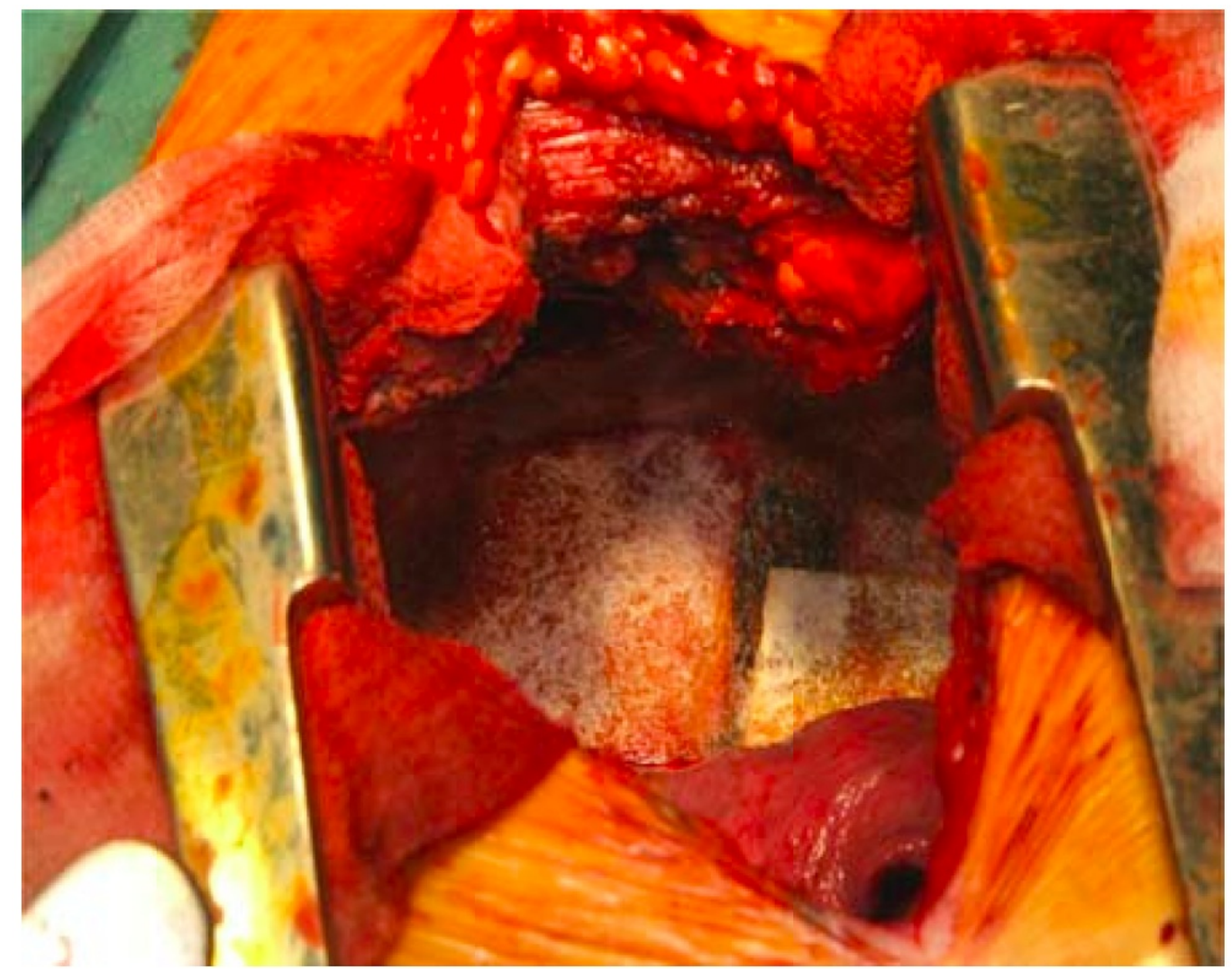

FIGURE 7: Intraoperative aspect. Hemostasis with TachoSil (paravertebral application)

Histopathological intraoperative examination revealed a cavernous capillary hemangioma, the findings of which were consistent with final paraffin-embedded microscopy (Figure 8). 


\section{Cureus}

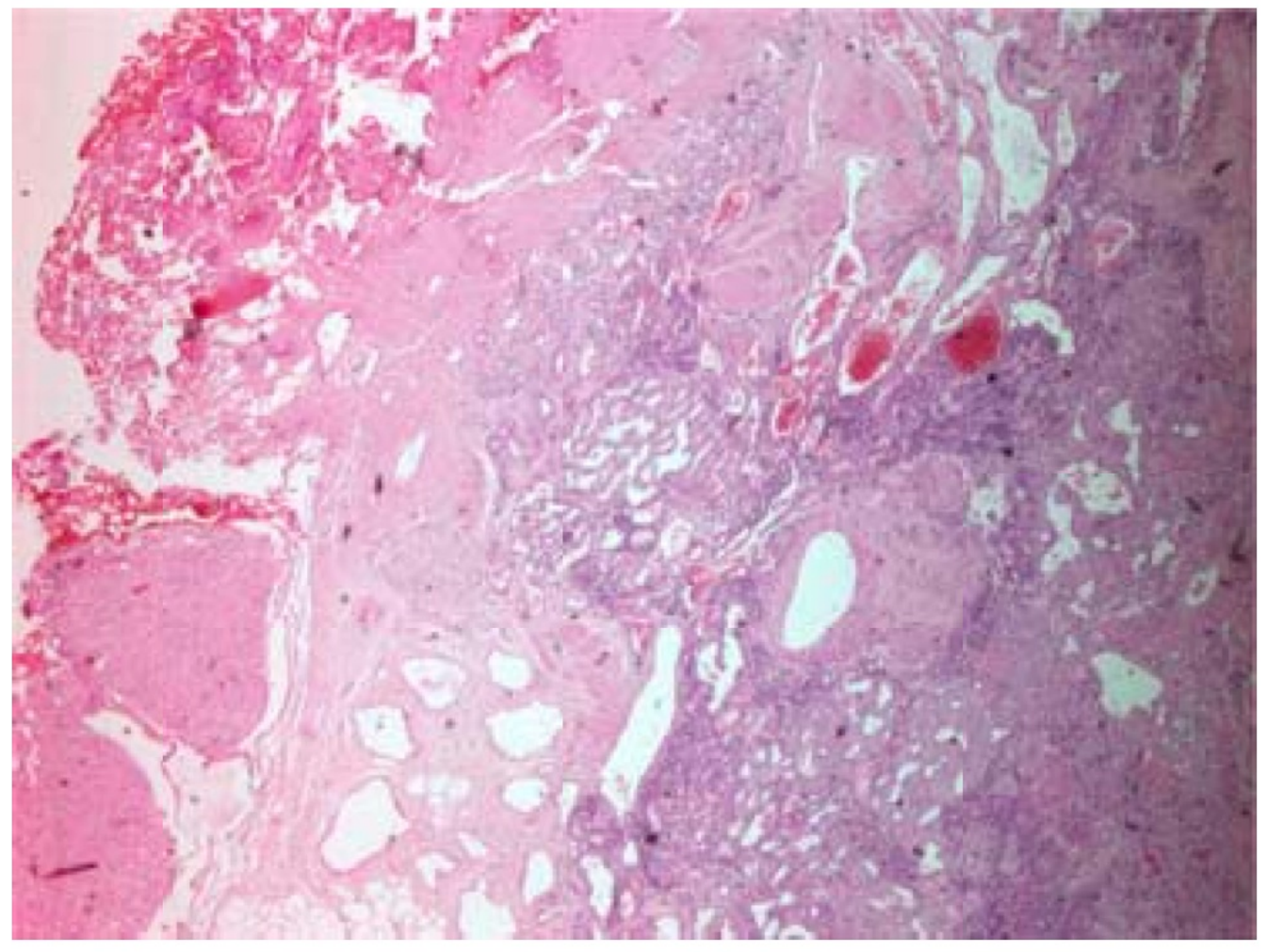

FIGURE 8: Capillary cavernous paravertebral hemangioma. Microscopic study (20xHE).

The lesion was positive for CD34 immunohistochemical markers (Figure 9) and actine (Figure 10) within vessel walls, confirming its benign nature and vascular origin, with limited proliferative tendency. 


\section{Cureus}

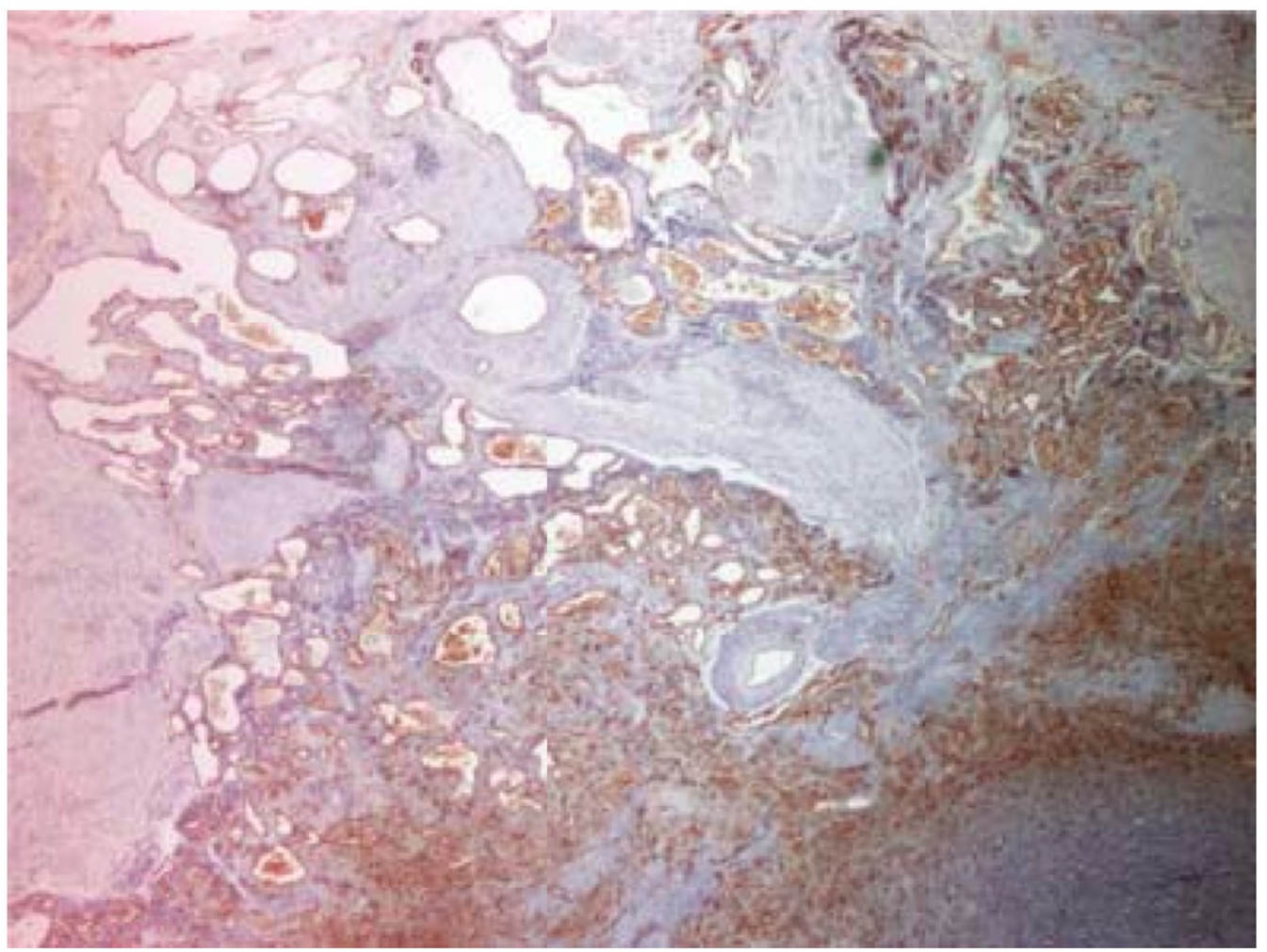

FIGURE 9: Immunohistochemical study. CD 34 marker pozitiv on vessels wall. (20xCD34)

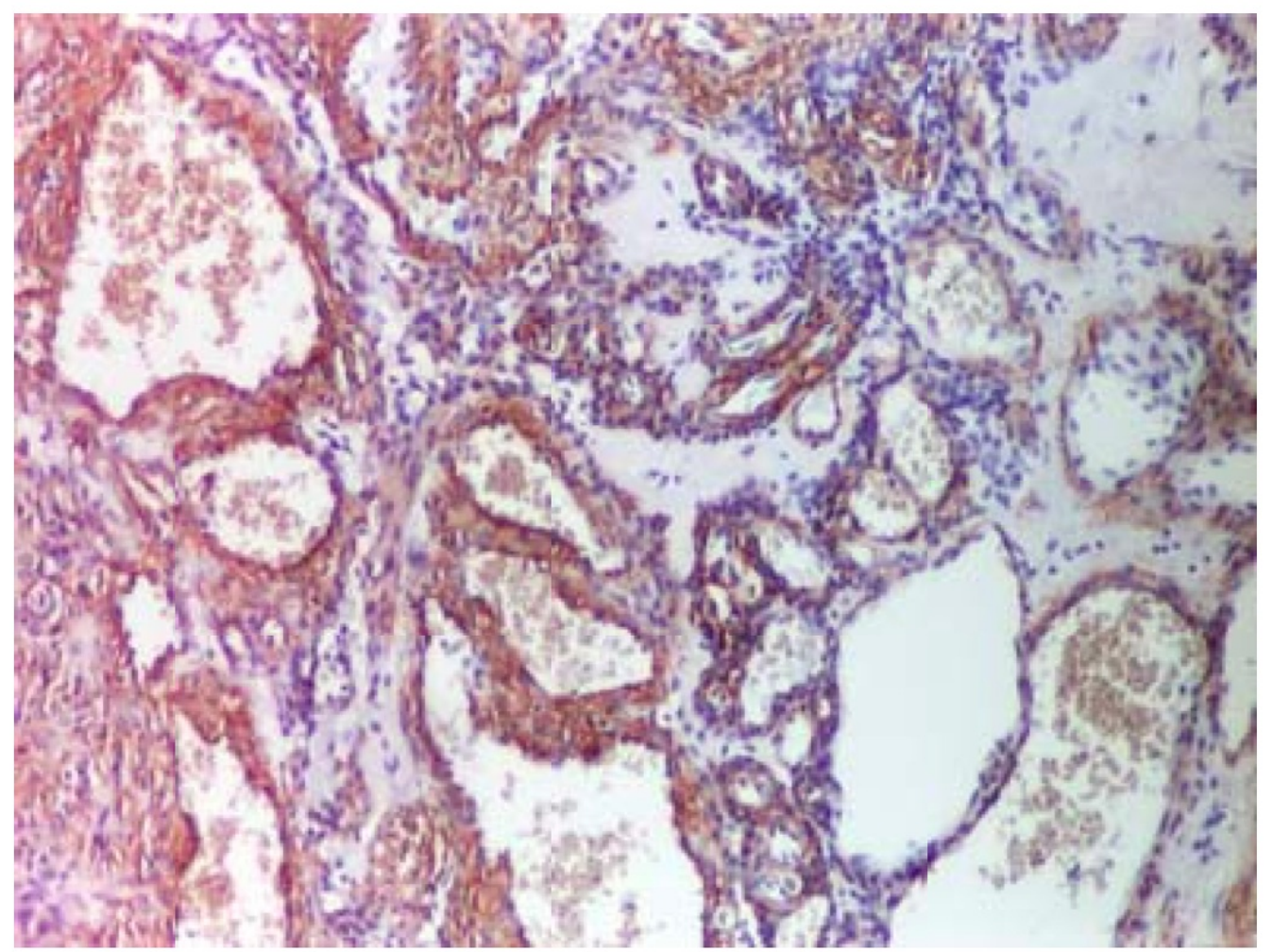

FIGURE 10: Immunohistochemical study. Actine positive on 


\section{Cureus}

vessels wall $(20 \times 0 B)$

Postoperatively, there was a good outcome with healing per primam and no evidence of tumor recurrence after two years. Postoperative MRI confirmed a significant resection of the lesion which over the ensuing two years remained stable in size and continues to respect the osseous boundaries of the adjacent veterbra (Figure 11A, 11B).

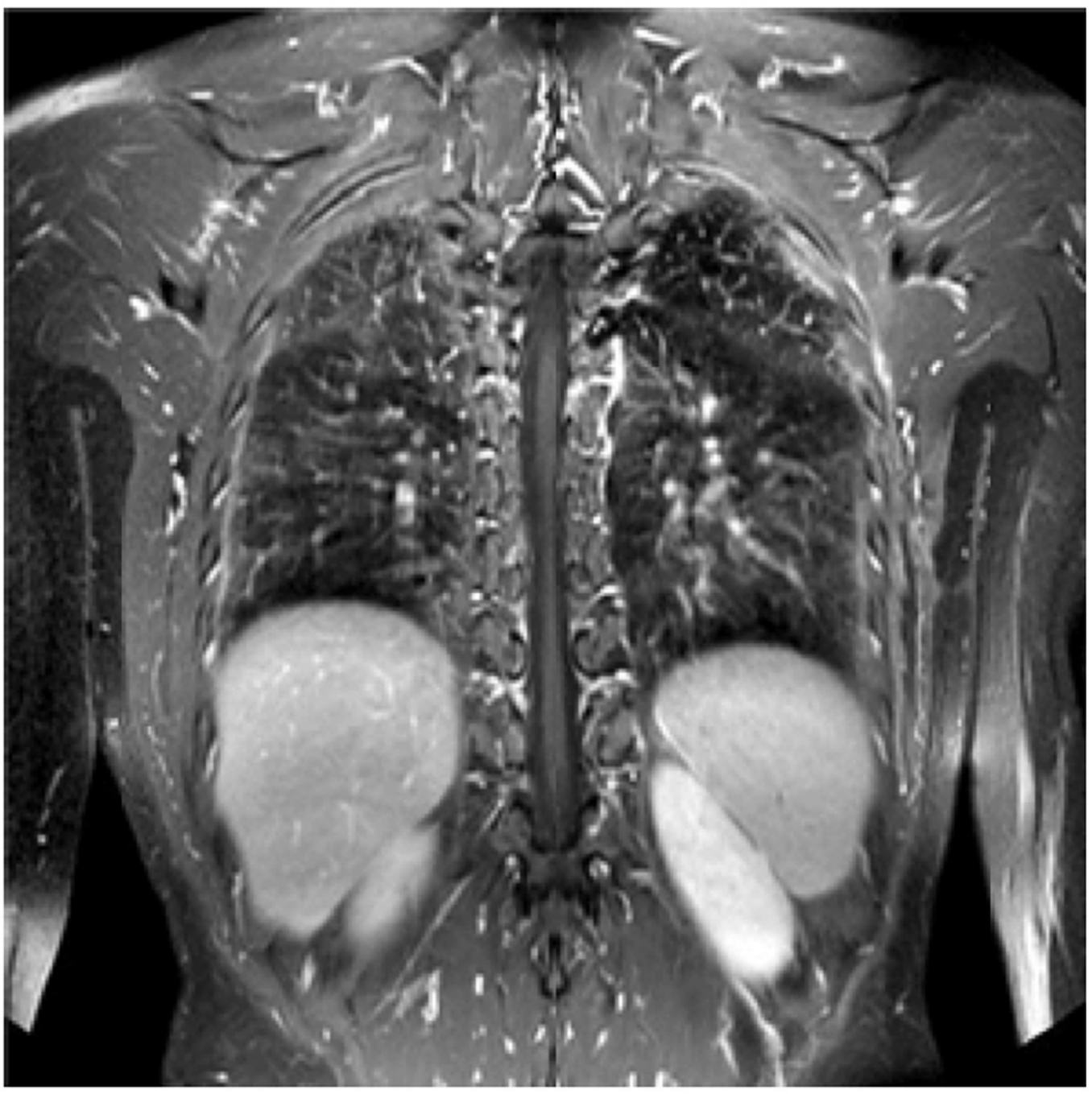

FIGURE 11: IRM aspect two years ago at postoperative care.

A. Paravertebral region after surgery without recidives B. Vertebral body T6 hemangioma with cortical bone limits.

\section{Discussion}

Hemangiomas are a relatively rare disease in adult pathology, estimated to account for about $0.5 \%$ of all mediastinal tumors [1]. The most frequently thoracic localizations are in the vertebrae, in the intercostal muscles, in subcutaneous tissue, skin, lung parenchyma, in the ribs, and in the posterior mediastinum, and in $25-30 \%$ of cases, they may be multiple [2]. Mediastinal localizations are very rare. One hundred cases have been communicated in the literature with the favored location being in the posterior and anterior-superior mediastinum 
From a nosological point of view, it has been asserted that hemangiomas are in a class with congenital arteriovenous malformation despite a tumor-like appearance (hamartoma) [4]. However, the modern perspective classifies hemangioma as benign neoplasia of a vascular origin that include neoformation vessels and have the potential to be locally destructive [3, 5]. Furthermore, recently reported chromosomal alterations, "mass" effect with compression of neighboring structures, and a macroscopic appearance of solid or mixed tumors also support the status of a "true neoplasia" [4].

Histopathologically, two main types of hemangiomas are found among thoracic lesions: 1) a cavernous type, frequently with significant expansion of ducts that store considerable quantities of blood and which contains vascular lakes, and a pseudo-capsule without vascular elements as well as fibrosis, post-thrombotic drainage and smooth muscle cells. Such lesions can have either a nodular or diffuse appearance and harbor a tendency for spontaneous regression [4], and 2) a capillary type with smaller neoformation vessels, fibroblasts, and few mitoses within endothelial cells, and which, in the pediatric age group, have a proclivity for spontaneous regression [4].

The pathogenic defect among hemangioblastoma is believed to center on a dysfunction of angiogenesis occurring during periods of blood vessel formation within primitive vascular networks: in the plexiform stage, when there is a network of capillaries that communicate, may occur capillary hemangiomas or in the retiform stage when exist big tubular vessels, with a tendency to coalescence, it forms cavernous hemangiomas and arteriovenous malformations $[4]$.

Although it appears that most hemangiomas in adults are asymptomatic, there are rare cases in which chest pain is the dominant symptom. Meanwhile, there are situations in which the clinical onset consists of severe complications that can even become surgical emergencies associated with massive haemopneumothorax, mediastinum perforation, and chest wall hematoma. Other serious symptomotology includes recurrent pleuresis and severe thrombocytopenia identified with Kasabach Merritt syndrome or with Maffuci syndrome. Paraplegia, spinal cord compression from hemorrhage, and progressive muscle weakness in cases of vertebral cavernous hemangiomas have also been reported in $15 \%$ of cases. Given the deceptive preoperative clinical picture that often mimics a malignancy, diagnosis of cavernous hemangiomas is difficult, and is often only resolved by histopathologic examination.

However, it is appreciated that diagnostic value of angio-MRI with three-dimensional study is compelling, especially for assessing invasion of the neighboring tissue, the degree of bone lysis, the spinal compression, and intra-thoracic development of the vertebral hemangiomas and is suggestive for differential diagnosis with other thoracic tumors, although, in the spinal capillary hemangioma, the selective arteriography has a superior value. Some authors recommend transthoracic ultrasound for differential diagnosis of chest wall tumors and for therapeutic purposes, for thoracentesis and percutaneous biopsy, and computed tomography scan, but MRI is the method of choice.

This case was, however, particularly difficult, because of the topography and structure with very rare capillary and cavernous combination of V-VI intercostal hemangioma, but also because of the synchronization with a T6 vertebral body hemangioma, with suggestive cavernous type appearance on MRI. A paravertebral location, sarcoma-like appearance on MRI, and the development along the intercostal space allowed differential diagnosis with paravertebral mesothelioma and neurinoma, although it was also possibly a chest wall hemangiosarcoma. 
However, the striking peculiarity of the presented case is the synchronism of those two hemangiomas situated in the T6 vertebral body and V-VI paravertebral space, a situation which allowed a differential diagnosis with "dumbbell shaped" tumor type, very rarely reported in the literature. But in this context, the two synchronous hemangiomas actually represent a "dumbbell shaped" lesion type?

Tumor invasion of the intervertebral foramen is often accompanied by a gradual painful radiculopathy which, in rare cases, can progress to paraplegia. Clearly, the imaging procedure of choice is MRI which best highlights the widening of the intervertebral space. Nevertheless, there are reported cases in which foraminal invasion could be definitely demonstrated intraoperatively, but which was invisible on preoperative MRI.

In the patient we present, there was no epidural or foraminal invasion, which is consistent with the absence of radiculopathy. Meanwhile, the MRI appearance of a cavernous hemangioma confined to the vertebral body, without breeching the cortical surface (Figure 3A, 3B), was confirmed intraoperatively (Figure o). Based on their topography, we presume that the paravertebral hemangioma represents an extra-thoracic extension of the vertebral lesion.

\section{Conclusions}

Thoracic hemangiomas are rare malignancies with vascular origin, which have misleading clinical-evolutionary aspects, requiring a difficult differential diagnosis. In terms of imaging, MRI with three-dimensional angiography, and sometimes arteriography, represent methods of choice for diagnosis. The differential diagnosis should include the arteriovenous malformations, malignant cancers, and, in the particular case of synchronous lesions with paravertebral topography, "dumbbell shaped" tumor type, because the treatment is different. The main treatment is surgical, based on the complete excision of the lesion, but there are complementary or alternative therapies, such as embolization, alcohol injection with spinal reconstruction, chemotherapy, corticosteroids, and anti-tumor immunotherapy. There is no standard treatment, but treatment options tailored to topography and evolutionary stage, especially in synchronous forms. The presented case is particular due to topography, histopathological structure, locally destructive nature, and "special type" synchronization, which requires a "particular" differential diagnosis and may represent a model for an interdisciplinary treatment approaches.

\section{Additional Information}

\section{Disclosures}

Human subjects: All authors have confirmed that this study did not involve human participants or tissue. Conflicts of interest: In compliance with the ICMJE uniform disclosure form, all authors declare the following: Payment/services info: All authors have declared that no financial support was received from any organization for the submitted work. Financial relationships: All authors have declared that they have no financial relationships at present or within the previous three years with any organizations that might have an interest in the submitted work. Other relationships: All authors have declared that there are no other relationships or activities that could appear to have influenced the submitted work.

\section{References}

1. Klecker Rosemary, Sinclair D, King M, Christoforidis AJ, Mueller CF: Chest: Case of the day. AJR. 2000, 175:866-871.

2. Hwang PM: Vertebral abnormality in a patient with suspected malignancy . Proc (Bayl Univ Med Cent). 2002, 15:325-326.

3. DeVita V, Hellman S, Rosenberg S: Cancer-Principles and Practice of Oncology . JB Lippincott 


\section{Cureus}

Company, Philadelphia; 1993.

4. $\quad$ Rosai J, Ackerman S: Surgical Pathology. Elsevier Inc, Amsterdam; 1993.

5. Hernigou P, Djindjian M, Ricolfi F, Dahhan P: Neuro-aggressive dorsal vertebral hemangioma and vertebrectomy. Apropos of 2 cases. Review of the literature. Rev Chir Orthop Reparatrice Appar Mot. 1994, 80:542-50 [Article in French]. 